STUDIA Z PRAWA WYZNANIOWEGO

Tom $23-2020$

DOI: https://doi.org/10.31743/spw.10355

GAETANO DAMMACCO*

\title{
RIFLESSIONI SUL DIRITTO DI SATIRA E I SUOI LIMITI
}

\author{
Riassunto
}

La satira è un paradigma estremo della libertà di espressione, ma esistono incertezze sulla sua definizione concettuale e sulla relativa disciplina giuridica. Lo sviluppo della comunicazione ha prodotto numerose figure letterarie simili tra loro come la cronaca (una registrazione impersonale e non interpretativa di fatti accaduti), la critica (analisi soggettiva e giudizio relativi a fatti accaduti) e la satira (critica sarcastica di personaggi, comportamenti, modi di fare individuali con scopo di denuncia sociale). Gli elementi che caratterizzano la satira, sviluppatisi nel corso dei secoli, sono sostanzialmente due: attenzione alle contraddizioni (della politica, della società, della religione, della cultura) e intento moralistico per promuovere un cambiamento sociale. La satira religiosa colpisce il potere ecclesiastico e le sue contraddizioni, ma colpisce anche i simboli religiosi e i contenuti delle religioni. Ne conseguono differenti conseguenze giuridiche. Quando colpisce il patrimonio di fede dei credenti essa non è accettabile. La satira religiosa genera una specie di conflitto tra differenti valori costituzionali, e cioè tra il diritto alla libera espressione del pensiero e il diritto alla reputazione e alla tutela del sentimento religioso. Il diritto di satira in generale è riconosciuto dagli ordinamenti giuridici (sia internazionali, sia nazionali) come diritto soggettivo di rilevanza costituzionale, che deriva dalla libertà di espressione e di pensiero. Pensiero, coscienza e religione - per esempio nella Carta dei diritti fondamentali dell'Unione europea - sono omologhi (come beni giuridici o valori etici). Pertanto pensiero, coscienza e religione non possono essere in contrapposizione tra loro. Notevoli incertezze esistono sulla disciplina giuridica del diritto di satira, che non può mai offendere i diritti

* Prof. dr. Università degli Studi di Bari Aldo Moro, Piazza Umberto I - 70121 Bari, Italia, e-mail: gdammacco48@gmail.com. ORCID 0000-0002-3452-4449. 
fondamentali della persona, la sua dignità, la sua reputazione. La Carta di Nizza ha favorito un orientamento, che considera il diritto di libera espressione nella sua forma più ampia ed espansiva. È tuttavia sempre stato affermato il valore prevalente dei diritti umani fondamentali, che non possono essere offesi dall'esercizio del diritto di satira. Negli ordinamenti giuridici nazionali, la forza del diritto di satira consiste nel riconoscimento del suo rango costituzionale, ma anche nei limiti che deve avere. La giurisprudenza ha elaborato i vincoli "formali", tra i quali i più importanti sono il limite della continenza e della funzionalità.

Parole chiave: satira; religione; diritti umani; limiti

$* * * * *$

\section{INTRODUZIONE}

Il 7 gennaio 2015 contro la sede del giornale satirico francese Charlie Hebdo (noto per il suo spirito caustico e irriverente) si consumò un mortale attacco terroristico ad opera di fondamentalisti dell'Isis, una tragedia assolutamente ingiustificabile e inaccettabile. Quell'episodio ripropone tra gli altri temi quello del diritto di satira, del suo contenuto, dei limiti che devono essere osservati. Il magazine sosteneva che la sua azione critica, coerentemente con lo stile adottato come scelta editoriale, aveva come obiettivo la difesa delle libertà individuali, civili e collettive, e al tempo stesso la difesa del diritto alla libertà di espressione. Tuttavia, proprio la gravità della tragedia e la sua influenza sulla opinione pubblica impone una più attenta analisi di natura giuridica come risposta agli interrogativi che la vicenda pone.

\section{NECESSITÀ DI UNA DEFINIZIONE DEI CONFINI CONCETTUALI: CRONACA, CRITICA, SATIRA}

La satira può essere considerata come un paradigma estremo della libertà di espressione, ma le incertezze sulla sua definizione concettuale e sulla relativa disciplina giuridica sovente ne fanno un paravento dietro il quale giustificare ogni tipo di dileggio e di offesa. Infatti, lo sviluppo 
della comunicazione ha prodotto numerosi strumenti e figure letterarie abbastanza simili tra loro come la cronaca, la critica e la satira, le quali costituiscono un forte veicolo comunicativo, perché ricostruiscono emozioni "relative a un certo periodo storico e a un certo contesto" tanto da condizionare il pensiero e la vita delle persone. È necessario, quindi, distinguere le diverse figure anche al fine di individuare nel modo migliore la disciplina giuridica idonea a tutelare i diritti corrispondenti (sebbene tutti riconducibili al diritto di libertà di espressione) e a stabilire il giusto e graduato riferimento con la verità.

\subsection{LA CRONACA}

La cronaca è in sostanza una registrazione impersonale e non interpretativa di fatti accaduti, dei quali chi scrive è stato in qualche modo testimone. Questi fatti sono registrati secondo la loro successione cronologica e con il riferimento ai soggetti protagonisti degli eventi, il più possibile rispondente alla verità degli accadimenti. Per l'importanza che i fatti di interesse pubblico assumono nella evoluzione dei contesti e delle relazioni sociali, la loro conoscenza genera un diritto a registrarli (nel modo più corretto possibile) e definisce un dovere corrispondente. Il diritto di cronaca è incluso nella maggior parte degli ordinamenti giuridici tra le libertà di manifestazione del pensiero (riconosciuto dalla Dichiarazione universale dei diritti dell'uomo del 1948 all'art 19, dalla Convenzione Europea sui diritti dell'uomo del 1950 all'art. 10 ora riversato nella carta di Nizza del 2000 all'art. 11 e dalla Costituzione italiana all'art 21) e per questo in generale la maggior parte degli ordinamenti democratici escludono l'imputabilità in colui che li riferisce (ad esempio gli articoli 50-54 del Codice penale italiano inseriscono il diritto di cronaca tra le cause di esclusione dell'imputabilità). Con riguardo al diritto di cronaca come diritto di informare e di essere informati è importante ricordare come, sin dalla sentenza n. 105 del 10 luglio 1972, la Corte Costituzionale italiana ha ritenuto che si tratta di diritti propri dei sistemi democratici i quali implicano la necessità "di una pluralità di fonti di informazione, libero accesso alle medesime, assenza di ingiustificati ostacoli legali, anche temporanei,

1 Pasquini 2012, 1. 
alla circolazione delle notizie e delle idee". Inoltre, la giurisprudenza ha ravvisato la necessità di porre dei limiti al diritto di cronaca al fine di bilanciare l'interesse alla libertà di informazione e l'interesse alla protezione della sfera personale. Dopo un lungo processo interpretativo, la Suprema Corte di Cassazione italiana (ad esempio, si possono considerare le sentenze: Cassazione penale del 30 giugno 1984, n. 8959 e Cassazione civile del 18 ottobre 1984, n. 5259) ha delineato le caratteristiche che il diritto di cronaca deve presentare in modo che la condotta del giornalista sia contenuta nell'ambito di limiti della legalità. Pertanto, è stata elaborata una nozione di limiti del diritto di cronaca che deve avere i requisiti di continenza, di pertinenza e soprattutto di veridicità dell'informazione, affinché l'esercizio del diritto di cronaca, a condizione che si tratti di fatti di pubblico interesse, possa prevalere sul diritto alla riservatezza e all'onore del protagonista della notizia.

\subsection{LA CRITICA}

Diversamente della cronaca, la critica consiste in una analisi soggettiva e in un giudizio relativi a fatti accaduti (di qualsiasi natura), purché i fatti rispondano sempre a un interesse pubblico. Questo genere letterario, quindi, si riferisce a una valutazione, che può avere giudizi di contenuto positivo oppure di contenuto negativo di fatti e avvenimenti. Così intesa, la critica è l'espressione più antica della libertà di pensiero (sotto il profilo filosofico, etico, religioso, estetico, politico), che nel corso dei secoli si è sviluppata sempre più verso un approccio critico diffuso, cioè non più riservato solo a poche persone (filosofi, pensatori, teologi, ecc.) ma condiviso da un pubblico sempre più ampio e sempre meno specialista; ciò ha consentito la nascita di un vero e proprio genere giornalistico. In particolare, nei secoli diciannovesimo e ventesimo le correnti di pensiero (storicismo idealista, sociologismo, marxismo, ecc.) si sono trasformate in strumenti diffusi di analisi, grazie all'incremento dei vari mezzi di comunicazione e più recentemente dei social, che hanno la forza di condizionare i comportamenti e le scelte delle persone. Un importante elemento di distinzione tra cronaca e critica consiste nel fatto che la critica contiene l'esternazione di un'opinione soggettiva, che per sua natura non deve essere coerente con la verità, pur restando agganciata alla verità dei fatti e, quindi, limitata da criteri di pertinenza e conti- 
nenza, e purché i fatti sui quali poggia la critica conservino un interesse pubblico, il loro profilo essenziale, non siano travisati e manipolati. Ciò significa che la ricostruzione di un fatto che si vuole criticare non deve contenere gravi omissioni o una ricostruzione che sconvolge la dinamica o il significato, poiché la critica di un fatto manipolato assumerebbe il valore di un giudizio falso e dannoso per il destinatario. Anche il diritto di critica trae la sua tutela dal diritto alla libera manifestazione del pensiero, come diritto rientrante nei diritti fondamentali della persona umana, generalmente accolti nelle carte costituzionali dei paesi democratici. Tuttavia, le espressioni usate nell'esercizio del diritto di critica devono essere strettamente funzionali alla manifestazione del commento critico, ma non di tale natura da degradare o screditare o ledere la reputazione altrui. Si tratta di garantire un bilanciamento degli interessi tra chi esercita il diritto di critica e il destinatario della critica, che ha interesse alla tutela della personale reputazione. In sostanza, le espressioni devono essere formalmente misurate in modo tale da non trascendere in attacchi e aggressioni personali diretti a colpire sul piano morale la figura del soggetto criticato (così ha deciso, ad esempio, in Italia il Tribunale di Milano sez. I, in una recente sentenza del 21 agosto 2018, n. 8738 , coerente con la precedente giurisprudenza in materia). In particolare, nell'esperienza italiana, la giurisprudenza della Corte di Cassazione (per ultimo si cita la Cassazione penale, sez. V, 23 gennaio 2017, n. 8482) ha esteso la tutela dell'onore a tutte le condotte offensive dell'altrui reputazione, realizzate con gli strumenti che veicolano la pubblicità, con i mass media, con i social networks, cioè con i moderni strumenti tecnologici che favoriscono la trasmissione di dati e di notizie a un numero elevato di persone. Una nota particolare deve essere fatta per quanto riguarda l'esercizio della critica basato sulla rigorosa osservanza di precetti religiosi o ideologici o pedagogici, poiché in questi casi c'è il rischio che fondamentalismo e intolleranza, che potrebbero essere anche giustificati da regimi totalitari, possano prevaricare il rispetto della persona e dei suoi diritti, e diventa pericolosa anche perché induce la popolazione alla assunzione di comportamenti violenti.

\subsection{LA SATIRA}

La satira è un genere di letteratura e di comunicazione, che nasce dalla necessità individuale o collettiva di porre in essere una attenzione parti- 
colamente critica alla politica, alla società, a personaggi influenti, mettendo in risalto anche con sarcasmo le contraddizioni presenti in espressioni, comportamenti, modi di fare individuali. Essa, come specifica la Corte di Cassazione italiana "costituisce una modalità corrosiva e spesso impietosa del diritto di critica. Inoltre ha lo scopo di denuncia sociale e politica. [...] quanto più [essa] utilizza espressioni abnormi, iperboliche, impietose, corrosive, esagerate rispetto ai normali parametri di valutazione degli esseri e delle cose umane, così da suscitare stupore, ironia, riso in colui che legge o ascolta". Anche nella letteratura latina la parola "satira", considerando l'origine del termine (derivante dal latino satura lanx, che era il vassoio vuoto riempito di primizie offerte agli dei), si riferiva a un genere letterario (prima teatrale poi solo poetico) che aveva l'obiettivo di mettere in ridicolo personaggi, ambienti, costumi con toni comici o sarcastici e con intenti moralistici (ad esempio sono famose le satire di Orazio), in qualche modo riempito di contenuti "spirituali".

Gli elementi che caratterizzano la satira e che si sono diversamente combinati nel corso dei secoli sono sostanzialmente due: attenzione alle contraddizioni (della politica, della società, della religione, dei comportamenti) e intento moralistico per promuovere un cambiamento sociale. La satira si è evoluta nei secoli, assumendo sempre più una funzione sociale e proponendosi come strumento di reazione. In particolare, durante l'illuminismo la satira fu molto usata contro i dogmatismi della religione e i privilegi dei nobili. Ad esempio, in questa direzione si collocano i lavori satirici di Voltaire (la più famosa opera è Candido), di Montesquieu (Lettere persiane), di Giuseppe Parini (Il Giorno) oppure le commedie di Beaumarchais, i libelli di Jonathan Swift e le 17 satire di Vittorio Alfieri). Nel Novecento, specie in corrispondenza dell'affermarsi dei totalitarismi, la satira non fu solo un genere letterario, ma propose anche un genere non letterario (si potrebbe dire popolare) che mescolava comicità e umorismo anche attraverso l'uso sempre più frequente di nuove tendenze dell'arte figurativa e dei nuovi media. La grande ecletticità del genere satirico di fatto ne sta cambiando le caratteristiche facendone un grande contenitore di strumenti di comunicazione (sia letterario sia non letterario), immediatamente percepibili dal grande pubblico, tendenti a ridicolizzare o a criticare con sarcasmo, con una vis comica maliziosa (e talvolta maligna), con spirito licenzioso, come espressione del pensiero di chi produce la satira e (non raramente) senza carica morale. 


\section{LA PECULIARITÀ DELLA SATIRA RELIGIOSA}

La satira religiosa occupa un posto particolare nella storia di questo genere di comunicazione e di letteratura. La vita delle persone nella società è sempre stata legata alla religione, che ha avuto notevoli ricadute nelle vicende culturali, economiche, politiche dei diversi sistemi sociali. Ciò ha comportato anche un rafforzamento della presenza "politica" (nel senso più ampio e in quello più stretto) della religione e del clero, che diventato spesso "attore sociale" è stato oggetto di satira. Vi è anche un secondo modo di fare satira religiosa, quando essa ha per oggetto i simboli religiosi e i contenuti (talvolta spirituali, ma più spesso culturali e tradizionali) delle religioni. Questi due modi di realizzare la satira religiosa comportano due differenti problemi giuridici: quando si tratta di satira che colpisce personaggi appartenenti al clero o alle varie autorità ecclesiastiche (volendo colpire la loro intransigenza oppure alcune prese di posizione su alcuni temi sensibili come ad esempio aborto, divorzio, oppure volendo censurare scandali "religiosi" di varia natura), si può intervenire contro la satira che supera il limite del rispetto dovuto alla persona umana; mentre, quando la satira riguarda i simboli delle religioni e il soggetto destinatario è la intera comunità religiosa (in modo diretto o indiretto), cioè un soggetto comunitario colpito nella sua dimensione esistenziale, diventa socialmente e giuridicamente inammissibile. In questo caso, la satira non ha un rapporto oggettivo con un dato reale (sia pure deformato o enfatizzato), ma riguarda il patrimonio di fede e il "credo" dei fedeli coinvolti nella esperienza di comunità, cioè il significato stesso della comunità religiosa. Sostanzialmente, la satira finisce per avere come oggetto il patrimonio religioso soggettivo, che si esprime attraverso i simboli che non appartengono a un grande pubblico, ma sono patrimonio nel quale il fedele ripone il significato stesso della sua esistenza. La caratteristica privata della fede, quindi, non giustifica l'esistenza di una satira che l'ordinamento ammette solo di fronte a un interesse pubblico e a una persona che svolga un ruolo pubblico.

Si deve considerare, inoltre, che la satira religiosa genera una specie di conflitto tra differenti valori costituzionali, e cioè tra il diritto alla libera espressione del pensiero (ad esempio, tutelato nell'art. 11 della Carta dei diritti fondamentali dell'Unione europea, nell'art. 21 della Costituzio- 
ne italiana e nell'art. 54.1 della Costituzione polacca) o il diritto alla libera espressione artistica (tutelato nell'art. 13 della Carta dei diritti fondamentali dell'Unione europea, nell' art. 33 della Costituzione italiana e nell'art. 73 della Costituzione polacca) da un lato e dall'altro lato il diritto alla reputazione e alla tutela del sentimento religioso (tutelato nell'art. 10.1 della Carta dei diritti fondamentali dell'Unione europea, nell'art. 53.2 della Costituzione polacca, nell' art. 19 della Costituzione italiana, norma che sancisce la libertà di religione).

In effetti, la satira religiosa non raramente è frutto della ignoranza circa le religioni (il loro contenuto, la loro funzione sociale e spirituale) e molto spesso chi produce la satira risponde a una reazione impulsiva risalente ad antichi conflitti. In particolare, specie in Italia, l'origine della satira religiosa si fa risalire alla tradizione carnevalesca popolare del rinascimento, che diede nuovi "contenuti" ai riti romani celebrati in onore di Saturno. Come è stato acutamente osservato, l'idea del carnevale rinascimentale "non è il godimento, l'ebbrezza dell'oggi, ma la mortificazione, la privazione del domani; non un inno ai sensi, alla carne, come ci aspetteremmo, ma un grido di dolore, di rimpianto, il grido dell'animalità insoddisfatta, stanca forse ma non sazia, la quale pensa che tutto quel godimento sta per finire"2. In questo contesto contraddittorio tra rigore e sfrenatezza, tra mortificazione ed ebbrezza trova spazio la critica impertinente, lo scherno, il ridicolo, l'attacco corrosivo alle passioni, alle istituzioni, al potere e ai loro detentori, ai modi di vita, ad atteggiamenti contrastanti con la morale, con la legge, con il sentire comune: prende vigore così il particolare e difficile rapporto tra satira e religione ${ }^{3}$. Si tratta di una relazione che facilmente può diventare di dubbio gusto e può anche trasformarsi nella violazione del diritto

2 E' interessante questa interpretazione di Clemente Merlo nel suo famoso saggio I nomi romanzi del carnevale (in Merlo 1934, 95-138; pubblicato prima in tedesco nel 1911, Die romanischen Benennugen des Faschings), che contrasta con altri giudizi, come ad esempio quello di Goethe, che nel febbraio del 1788 è a Roma e descrive il Carnevale (lo scritto confluirà nel famoso Viaggio in Italia) come una festa che "il popolo si concede", quasi come una continuazione in altra forma degli svaghi domenicali e festivi. Più in generale sul carnevale vedi Kezich 2019.

3 Una riflessione su questo delicato rapporto e sugli effetti della satira religiosa nelle varie società nella ricerca di un equilibrio tra la libertà di espressione ed il rispetto per la fede altrui è in Bongiovanni 2018. 
di libertà religiosa (sia individuale sia al livello delle comunità religiose) e nella commissione di reati, come ad esempio quello di vilipendio della religione e di violazione delle norme a tutela del sentimento religioso.

La satira religiosa per il suo stesso peculiare carattere è sottoposta a limiti che derivano dal rispetto del buon costume, del comune sentire religioso nell'ambito della società, e anche dal rispetto di quella particolare forma del sentimento religioso che appartiene al sentire dei fedeli. Ciò significa che vi è un limite che è tipico del sentire etico sociale (e in questo non differenzia la satira religiosa dal contenuto di altre forme di satira come quella culturale, politica, ecc.) e un limite che è dato dalla particolare sensibilità dei fedeli (e in questo si differenzia dalle altre forme di satira). Senza dubbio si tratta di confini non chiaramente segnati e che possono cambiare in relazione alle altre variabili socio-culturali (tradizione, cultura, sensibilità, visione della vita, ecc.). Inoltre, il rapporto tra satira e religione è anche caratterizzato dal cambiamento di alcuni aspetti strutturali che derivano dal carattere sempre più plurale e multiculturale di una società che tende a concepirsi nella sua diversità e differenziazione, cosa che contribuisce a rendere sempre più incerto il loro confine. Del resto, sulla definizione concettuale di satira religiosa incide anche l'autocomprensione della sua esperienza religiosa da parte del fedele (più o meno rigida, più o meno consaspevole), il quale, lungi dall'essere un appartenente a uno schieramento ideologico o politico, orienta la propria personalità e la propria vita in base a un codice (la religione) che segna il suo stesso essere. Per questo, facilmente la satira rischia di realizzare una profonda mancanza di rispetto per la persona umana (toccata in quanto tale in un bene, come la religione, che conferisce alla propria esistenza senso e significato) e, conseguentemente, di entrare in quell'ambito che l'ordinamento giuridico considera sanzionabile. Inoltre, il legame tra satira e religione oggi deve anche tener conto dei cambiamenti strutturali della nostra società, nella quale il carattere del multiculturalismo sta assumendo una significativa rilevanza. Ciò contribuisce a rendere più difficile la linea di confine tra la satira e l'offesa sarcastica, poiché incidono le differenze culturali che comportano differenze nella sensibilità religiosa, nella diversa percezione dell'altrui manifestazione ironica e in una differente reazione alle ripercussioni che la satira può avere su ciò che definisce la persona e il valore delle sue espressioni di vita religiosa. Senza dubbio, la difficoltà a tracciare i confini tra il diritto di satira e il diritto di li- 
bertà religiosa, come scelte personali di vita, rende anche difficile l'elaborazione di una legge attraverso la quale stabilire in maniera incontrovertibile, anche tenuto conto di tutte le variabili introdotte dal multiculturalismo, quando la libertà di satira non è offensiva e quando invece essa è sanzionabile dall'ordinamento. Questa particolare condizione, rende ancor più importante l'intervento dei giudici, i quali dovranno giudicare tenendo conto non solo di quanto stabilito e previsto dalle leggi ma anche delle caratteristiche socio-culturali del momento in cui i fatti si sono realizzati.

\section{IL DIRITTO DI SATIRA COME DIRITTO SOGGETTIVO}

Il diritto di satira in generale è riconosciuto dagli ordinamenti giuridici come diritto soggettivo di rilevanza costituzionale, poiché attiene direttamente alla libertà di espressione e di pensiero. La Carta dei diritti fondamentali dell'Unione europea disciplina la libertà di pensiero nell'articolo 10 e la libertà di espressione nell'articolo $11^{4}$. La particolare formula dell'art. 10 consente di considerare il pensiero, la coscienza e la religione come appartenenti a un insieme di omologhi (considerati come beni giuridici, come obiettivi, come valori etici) in modo tale che pensiero, coscienza e religione non possono essere considerati in contrapposizione tra loro, nemmeno concettuale, e di conseguenza la loro tutela giuridica non può prevedere la prevalenza di uno sugli altri. In altri termini non si può immaginare di tutelare la libertà di pensiero contro la libertà di religione oppure la libertà di coscienza contro quella di pensiero. Se, dunque, il diritto di satira appartiene all'ambito della libertà di pensiero, è evidente che nella prospettiva e nella tutela dell'art. 10 non vi può essere contrap-

4 Così recita l'articolo 10: “1. Ogni individuo ha diritto alla libertà di pensiero, di coscienza e di religione. Tale diritto include la libertà di cambiare religione o convinzione, così come la libertà di manifestare la propria religione o la propria convinzione individualmente o collettivamente, in pubblico o in privato, mediante il culto, l'insegnamento, le pratiche e l'osservanza dei riti". Il successivo articolo 11 così recita: "1. Ogni individuo ha diritto alla libertà di espressione. Tale diritto include la libertà di opinione e la libertà di ricevere o di comunicare informazioni o idee senza che vi possa essere ingerenza da parte delle autorità pubbliche e senza limiti di frontiera. 2. La libertà dei media e il loro pluralismo sono rispettati". 
posizione tra la libertà di pensiero e la libertà di religione, anzi deve esserci compatibilità e unità di base interpretativa. Conseguentemente, non può esservi disprezzo verso la religione nell'esercizio del diritto di satira e laddove al contrario ciò accadesse la satira non può essere considerata tale e deve essere sanzionata non potendosi considerare esistente il corrispondente diritto.

Il contenuto dell'art. 10 della Carta europea corrisponde al contenuto dell' art. 9 della CEDU, in connessione di cui deve essere interpretata e applicata. Il paragrafo 2 dell'art. 9 stabilisce che il diritto di religione e di credo possono essere sottoposti solo a restrizioni previste dalle leggi, ciò a maggior tutela dei diritti di libertà e per evitare ogni forma di abuso, restrizione e discriminazione. Poiché in detto secondo paragrafo non è considerata la libertà di pensiero, che comprende anche la libera manifestazione del pensiero con ogni forma e strumento pubblico e privato (comprendendovi, quindi, anche la satira), resta da chiedersi se, alla luce delle formule giuridiche sopra indicate, la libertà di pensiero non sia sottoposta ad alcuna restrizione o se, al contrario, essa sia sottoposta a restrizioni che non provengono dalla legge, ma per esempio dalla attività amministrativa della pubblica amministrazione, cosa che in qualche modo sarebbe ancor più grave. Tuttavia, un intervento restrittivo autonomo della pubblica amministrazione sembrerebbe escluso dal contenuto del paragrafo 1 dell'articolo 11, che corrisponde all'art. 10 della CEDU e che esclude ogni forma di "ingerenza da parte delle autorità pubbliche", intendendosi per ingerenza ogni intervento arbitrario o abusivo in questioni sulle quali non è riconosciuta una specifica competenza. Tuttavia, non pochi sono gli ambiti nei quali la pubblica amministrazione giustifica un proprio intervento, basato sulla legge, come ad esempio gli interventi per questione di ordine pubblico. Se ammettessimo che dalla semplice lettura degli articoli 10 e 11 non risulta nessuna forma di restrizione normativa per la libera espressione del proprio pensiero attraverso la satira, dovremmo concludere che la satira non è sottoposta a nessuna restrizione e l'esercizio di tale diritto resterebbe limitato solo dalle specifiche norme penali che tutelano la persona e la sua dignità. Per altro aspetto, se ammettessimo che la pubblica amministrazione possa comunque intervenire, giustificando il proprio intervento sulla necessità di far rispettare le leggi di fronte a un pericolo (basterebbe ad esempio il pericolo per ordine pubblico, per motivi sanitari o per ga- 
rantire i pubblici servizi) lo spazio della discrezionalità sarebbe così ampio e incerto da reprimere un diritto di libertà.

Come si può notare, sotto il profilo normativo esistono notevoli incertezze, probabilmente dovute alla complessità della materia e al fatto che risultano coinvolte differenti posizioni personali e tutelati differenti diritti (soggettivi e pubblici) in capo ai diversi soggetti in gioco. Considerando anche l'evoluzione del genere "satira" e quanto impercettibile sia il confine con i diritti fondamentali della persona e con gli altri diritti di libertà (come quello di religione, che riguarda non solo le posizioni individuali, ma anche quelle confessionali), il ricorso all'azione dei giudici (sia delle corti internazionali sia di quelle nazionali) si è rivelato importante perché ha contribuito a fare qualche chiarezza. Con l'entrata in vigore della Carta dei diritti fondamentali dell'Unione europea, un orientamento giurisprudenziale della Corte Europea di Giustizia si è andato consolidando nel tempo, favorendo la considerazione del principio della libertà di espressione nella sua forma più ampia ed espansiva ${ }^{5}$. La Corte europea dei diritti dell'uomo e la Corte europea di giustizia si sono occupate più volte delle questioni derivanti dal diritto di satira e dei suoi limiti. In particolare, la Corte di giustizia dell'Unione europea ha rinviato sempre più spesso alla Carta, tanto che è stato calcolato che il numero di sentenze che motivano rinviando ai principi della Carta nel 2011 sarebbe aumentato di più del 50\% rispetto al 2010. Anche le giurisdizioni nazionali hanno più spesso motivato le sentenze con riferimenti alla Carta anche quando si trattava di questioni poste alla Corte di giustizia come ad esempio le questioni pregiudiziali (fra il 2010 e il 2011 tali riferimenti sono aumentati del 50\%, da 18 a 27). La crescente importanza dei diritti umani e della loro tutela è dovuto anche alla strategia adottata dalla Commissione europea nell'ottobre del 2010 per garantire l'effettivo rispetto della Carta (COM-2010-573), elaborando una "check-list dei diritti fondamentali" per agevolare la valutazione del loro impatto su tutte le proposte legislative. La Commissione si è inoltre impegnata a pubblicare una relazione annuale con la quale monitorare i progressi realizzati nella applicazione della Carta, cosa che è stata fatta per la prima volta il 16 aprile 2012 relativamente all'anno 2011 (COM(2012)169). La Relazione, nell'illustrare le iniziative assunte dalle istituzioni europee nel corso del

5 Sul punto cfr. Nascimbente, Rossi dal Pozzo 2019, 135-138. 
2011 in applicazione dei principi della Carta (comprese quelle per la parità tra donne e uomini 2010-2015), con specifico riferimento alla crisi economica in atto all'epoca, sostenne il valore del rispetto dei diritti fondamentali in un contesto giuridicamente stabile basato sull'idea dello stato di diritto come migliore garanzia per instaurare un clima di fiducia e di sicurezza nei cittadini e negli investitori. Il percorso di rafforzamento dei diritti umani fondamentali trova anche un punto di forza nell'impegno del Consiglio europeo, il quale non mancò di sottolineare l'importanza di includere nella Carta europea dei diritti fondamentali i principi generali sanciti dalla Convenzione europea dei diritti dell'uomo del 1950, quelli risultanti dalle tradizioni costituzionali comuni dei paesi dell'UE, i diritti fondamentali attribuiti ai cittadini dell'UE, i diritti economici e sociali enunciati nella Carta sociale del Consiglio d'Europa e nella Carta comunitaria dei diritti sociali fondamentali dei lavoratori, i principi derivanti dalla giurisprudenza della Corte di giustizia e dalla Corte europea dei diritti dell'uomo. In tal modo, la Carta europea (che poi fu sottoscritta a Nizza nel 2000) risulta (secondo gli auspici) un solido punto di arrivo di un cammino pluriennale compiuto a livello istituzionale da numerosi soggetti, ma allo stesso tempo rappresenta il punto di raccordo strutturale e ontologico tra i principi ivi contenuti e i principi costituzionali dei Paesi aderenti al Consiglio e all'Unione. Pertanto, lo spazio riconosciuto alla satira come materializzazione del diritto che tutela la libertà di pensiero e di espressione si può comprendere meglio proprio considerando l'intensità del cammino compiuto. Con riferimento a questo complesso cammino, più solida appare sia la tutela del diritto di libera espressione sia la tutela dei diritti personali fondamentali. Infatti, il contenuto del diritto garantito al paragrafo 1 dell'art. 10 della Carta dei diritti fondamentali dell'Unione nella corrispondenza con il diritto garantito dall'articolo 9 della CEDU, considerando il contenuto dell'articolo 52 paragrafo 3 della Carta dei diritti fondamentali, risulta avere un significato e una portata più ampia. Allo stesso modo, le limitazioni al diritto di satira derivano dal contenuto dell'articolo 9 paragrafo 2 , che, nel rispetto delle tradizioni costituzionali nazionali, tutela "la libertà di professare la propria religione o il proprio credo" in una società democratica, ponendo solo il limite della legge fondato su misure necessarie "alla pubblica sicurezza, alla protezione dell'ordine, della salute o della morale pubblica, o alla protezione dei diritti e della libertà altrui". 
Per quel che riguarda l'ordinamento italiano, il diritto di satira rientra nell' ambito applicativo degli articoli 21, 9 e 33 della Costituzione, che tutelano rispettivamente la libertà di pensiero e di espressione, lo sviluppo della cultura e la libertà di creazione artistica. Trattandosi di un diritto di rango costituzionale, l'esercizio di tale diritto è in qualche modo l'espressione convergente di una tutela di un bene pubblico (come può essere proprio la denuncia sociale o politica o religiosa perseguita) realizzata da un privato legittimamente. Infatti, in alcune sentenze viene enfatizzata la "non trascurabile funzione di moderare i potenti, smitizzare ed umanizzare i famosi, umiliare i protervi, vale a dire una funzione fondamentale di controllo sociale e di protezione contro gli eccessi del "potere", nonché di attenuazione delle tensioni sociali e di tutela ed attuazione del valore fondamentale della tolleranza" (Tribunale di Roma, 13 febbraio 1992).

\section{I LIMITI DEL DIRITTO DI SATIRA}

Tuttavia, la forza del diritto di satira non consiste solo nel riconoscimento del suo rango costituzionale, ma (come per ogni diritto) anche nei limiti che esso deve avere. Tra questi limiti deve essere contemplato prima di tutto l'esclusione di un conflitto tra diritti dello stesso rango costituzionale, quindi non risulta possibile un conflitto tra il diritto di satira e i diritti fondamentali della persona umana. D'altra parte, i limiti della satira (di qualunque natura essa sia, religiosa, politica o sociale) definiscono in qualche modo anche i doveri ai quali deve attenersi il suo autore, il quale, nell'esercizio del suo diritto, deve tenere una condotta che non si risolva in un'aggressione gratuita e lesiva dell'onore e della reputazione del soggetto interessato dalla critica satirica (così il Tribunale di Roma, sez. I, 1 dicembre 2017, n. 22585) e che sia rispettosa del bilanciamento dei diritti personali in gioco.

La giurisprudenza ha elaborato i vincoli "formali" riguardanti la struttura del genere satirico, che pur essendo sottratto al parametro della verità (cioè della corrispondenza tra satira e $\mathrm{i}$ fatti accaduti) non può superare il limite della continenza e della funzionalità delle espressioni o delle immagini rispetto allo scopo di denuncia (sociale o politica) perseguito. A questi si aggiungono i limiti della dimensione pubblica del soggetto colpito e della 
pertinenza, dovendo la satira presentare un nesso causale tra la popolarità pubblica del soggetto colpito e il messaggio morale di denuncia sociale, che l'autore vuole trasmettere al pubblico. Accanto a questi limiti formali, che poggiano sui principi contenuti nella Carta dei diritti fondamentali dell'Unione europea (ma anche sui diritti della CEDU), ci sono i limiti giuridici individuati dalle Carte costituzionali, come ad esempio il buon costume e la tutela dell'ordine pubblico, in modo che il bilanciamento di interessi sia concretamente realizzato.

Una menzione particolare merita un altro limite peculiare relativo alle espressioni di odio razziale e xenofobo. Come è noto, il contrasto a questo tipo di odio è una strategia di lotta dell'UE, che monitora come sia stata recepita nei Paesi membri la decisione quadro 2008/913/GAI sulla lotta contro talune forme ed espressioni di razzismo e xenofobia, anche secondo le indicazioni contenute in altra direttiva (la 2000/43/CE), che attua il principio della parità di trattamento fra le persone, indipendentemente dalla razza e dall'origine etnica. Questo limite riguarda specialmente Ebrei e Rom, i quali rientrano nel programma di promozione dell'integrazione sociale ed economica a seguito della comunicazione della Commissione “Quadro dell'UE per le strategie nazionali di integrazione dei Rom fino al 2020” (COM(2011)173).

La tutela del diritto di satira ha compiuto importanti passi anche grazie all'opera dei giudici (sia nazionali sia europei), sebbene restano ancora molti punti da chiarire in tema di esercizio della giurisdizione e alcuni derivano dalla particolare condizione che l'UE ha riconosciuto ad alcuni Paesi membri in sede di sottoscrizione dei Trattati europei. È un esempio il protocollo n. 30, allegato al Trattato di Lisbona, il quale prevede misure specifiche per il Regno Unito (oramai non più interessato dopo la "brexit") e per la Polonia, riconoscendo limitazioni alla giurisdizione della Corte di giustizia europea e anche a quella dei tribunali nazionali in tema di materia di conformità della normativa nazionale rispetto ai diritti, alle libertà e ai princìi sanciti dalla Carta. Infatti, il protocollo prevede che se una disposizione della Carta faccia riferimento a leggi e pratiche nazionali, essa si applicherà alla Polonia solo nella misura in cui i princìi e i diritti della Carta siano riconosciuti nelle leggi e nelle pratiche interne al Paese. Ciò comporta che se non esiste un atto di recepimento delle singole disposizioni della Carta, questa non si applicherà all'interno del Paese. Conseguente- 
mente, quando si tratta di libertà di espressione (anche attraverso la satira), diritto garantito dall'art. 11 paragrafo 1 della Carta dei diritti fondamentali dell'Unione europea, se non vi è un atto interno di accoglimento il diritto potrebbe non essere protetto all'interno della Polonia, con conseguenze facilmente immaginabili. Sulla base delle sentenze dei giudici europei, quindi, è possibile individuare i requisiti che deve avere la satira e a quali limiti essa deve essere assoggettata. È evidente che ogni fattispecie che non rispetti i canoni giuridici, frutto della applicazione interpretativa delle disposizioni europee e specialmente della Carta dei diritti, non può essere catalogata come satira e non può godere del particolare regime di tutela.

\section{LA SITUAZIONE IN ITALIA E L'INTERVENTO DEI GIUDICI}

Per quanto riguarda la situazione in Italia, le sentenze dei giudici si basano su motivazioni sostanzialmente conformi ai principi prodotti nel contesto europeo. Secondo numerose pronunce della Suprema Corte di Cassazione, la satira è una "forma artistica che fa uso dell'ironia fino al sarcasmo, essa si esercita da sempre nei confronti del potere, politico o economico, costituito". Ne consegue che la satira deve colpire prima di tutto il potere e le sue criticità e deve esser utilizzata per criticare le persone potenti, non deve essere utilizzata per offendere i deboli o chi è già colpito da varie gravi problematiche. Essa, pertanto, deve essere uno strumento di denuncia del malcostume della società, della politica, dell'economia, della cultura, della religione e non può essere un pretesto per giustificare un insulto gratuito o un giudizio denigratorio verso una comunità o una persona, anche quando si tratti di persona "poco rispettabile". Insomma, secondo la giurisprudenza, la satira (come critica "la più tagliente, incisiva e suggestiva") svolge la sua funzione sociale se induce a riflettere e non solo a indignare; e comunque "non deve offendere perché non può infrangere il rispetto dei valori fondamentali della persona umana" (così la Cassazione, sezione civile, nella sentenza n. 5499 del 2014). In ogni caso, la satira non può travalicare il limite della correttezza del linguaggio e del rispetto della dignità umana, cosa che si verifica quando il linguaggio e le espressioni usate si traducono in una specie di libero insulto (in questo modo, ad esempio, ha giudicato il Tribunale di Trento, in una sentenza del 26 gennaio 1999). Inoltre, come ha ancora sottolineato la giurisprudenza del- 
la Cassazione (sia civile sia penale), l'utilizzo di espressioni "forti" di qualsiasi tipo, "anche lesive della reputazione altrui deve essere strumentalmente collegato alla manifestazione di un dissenso ragionato dall'opinione o dal comportamento preso di mira e non deve risolversi in un'aggressione gratuita e distruttiva dell'onore e della reputazione del soggetto interessato", anche quando si tratti di soggetto giuridico ${ }^{6}$. In verità, circa l'uso di un linguaggio sarcastico e irriverente, la giurisprudenza non è univoca, poiché una parte sostiene che la satira, per la natura di espressione artistica caratterizzata da un linguaggio non convenzionale, non è obbligata alla correttezza dell'esposizione, mentre un'altra parte prevalente ritiene comunque che anche alla satira debba essere applicato il limite della continenza, sebbene questo sia un parametro difficile da valutare. Comunque, le espressioni, di qualunque natura esse siano, nel linguaggio della satira devono rispettare una relazione strumentale con la "manifestazione di un dissenso ragionato dall'opinione e dal comportamento preso di mira", essendo esclusa la mera ed ingiustificata denigrazione. Quando la satira è diffamante (ciò costituisce una variante della diffamazione a mezzo stampa) sussiste il diritto del soggetto a pretendere che la pubblicazione delle proprie vicende personali (specie se si tratta di fatti del passato) possa effettuarsi solo "quando sussista un interesse effettivo e attuale alla loro diffusione" da verificare di volta in volta (in tal senso, ad esempio, sono le sentenze della Corte di Cassazione n. $13161 \mathrm{del}$ 24 giugno 2016, n. 16111 del 26 giugno 2013 e n. 5525 del 5 aprile 2012).

Sulla base della giurisprudenza, quindi, è possibile individuare le caratteristiche che deve avere una satira, perché sia riconosciuto nel suo autore il diritto conseguenziale. Seguendo le decisioni della Cassazione, allineata con quanto stabilito dalle Corti europee, l'intero quadro normativo e giurisprudenziale consente di individuare le caratteristiche che deve presentare il genere "satira" e che possono così essere individuate: 1) la satira

6 In tal senso sono l'ordinanza della Cassazione n. 21235 del 17 settembre 2013 e le sentenze della Cassazione n. 1753 dell' 8 febbraio 2012 e n. 28411 del 28 novembre 2008. Di particolare interesse è la sentenza n. 54990 del marzo 2014, che risolve una controversia tra Codacons (associazione a tutela dei consumatori) e la Editrice Romana spa, editore del quotidiano "Il Tempo", in cui erano apparsi tre articoli di stampa che usavano un linguaggio offensivo e di disprezzo della associazione. In quella sentenza la Cassazione ha ribadito il principio sopra riportato. L'interesse è dato dal fatto che la decisione della Corte suprema ha inteso proteggere la dignità non di una persona fisica, ma di una persona giuridica. 
deve essere un contributo soggettivo a un dibattito di interesse pubblico, favorito dalla diffusione di immagini o di notizie; 2) l'interesse satirico alla diffusione dell'immagine o della notizia deve essere effettivo e attuale (per ragioni di giustizia, di tutela dei diritti e delle libertà altrui, per scopi scientifici, didattici o culturali, ecc.) e deve tener conto della dimensione pubblica del soggetto colpito e del "beneficio" etico e morale che può derivare alla società e ai suoi costumi; 3) L'interesse pubblico manca nel caso in cui prevalga un interesse privato alla divulgazione o un interesse meramente economico o commerciale; 4) deve essere presente un elevato grado di notorietà del soggetto (persona fisica o giuridica) colpito dalla satira per la peculiare posizione occupata nella vita pubblica; 5) il diritto di satira è diritto soggettivo di rango costituzionale, tutelato dall'art. 21 della Costituzione come libera manifestazione del pensiero oltre che dall'art. 9 e dall'art. 33; 6) Il diritto di satira ha un fondamento nella sua dimensione relazionale come messaggio sociale, esercitato con ironia e sarcasmo nei confronti dei poteri di qualunque natura; 7) la satira (in qualunque forma espressa) è una critica corrosiva basata non sulla verità dei fatti, ma sulla loro rappresentazione deformata, non è sottoposta al parametro della verità, ma al parametro della continenza e della funzionalità delle espressioni adoperate rispetto allo scopo di denuncia sociale perseguito; 8) essendo una forma particolare di comunicazione, è opportuno che la satira sia preceduta da una preventiva informazione in modo da consentire all'interessato il diritto di replica; 9) la satira non può mai tradursi in una offesa alla dignità della persona e ai suoi diritti umani fondamentali. Da questa esposizione sommaria discende la necessità che la satira sia valutata in concreto e tenendo conto di tutti gli elementi, poiché per individuare il genere satirico non basta che il suo autore definisca la manifestazione del suo pensiero come satira, ma è necessario che essa contenga tutti gli elementi oggettivi (tra i quali i più importanti sono stati sopra individuati) idonei a qualificarla come tale.

\section{CONCLUSIONI}

L'esposizione argomentata di quanto precede, può consentire di esprimere un giudizio più meditato sul fatto dal quale è partita la presente riflessione e cioè sulle vignette che il magazine Charlie Hebdo ha lasciato 
che fossero definite satiriche con riguardo alla religione islamica. Nel caso specifico, restano seri dubbi sul fatto che quella pubblicazione possa essere considerata come satira, poiché l'esercizio del diritto di satira finisce per ledere illecitamente altri diritti umani fondamentali. Infatti, essa contiene elementi offensivi di una religione (e non di specifici personaggi) e non sembra rispettare il limite della pertinenza, poiché non si vede un collegamento tra il giudizio satirico e il fatto, inoltre il "linguaggio" usato non è rispettoso dei valori religiosi e del sentimento religioso della comunità religiosa colpita. Infine, non risulta con chiarezza la manifestazione di un dissenso con l'opinione o il comportamento del soggetto preso di mira, con l'intenzione di fare una denuncia sociale.

\section{BIBLIOGRAFIA}

Bongiovanni, Ambrogio (a cura di). 2018. Satira e religioni, L'ironia salverà il mondo? Trapani: Il pozzo di Giacobbe.

Kezich, Giovanni. 2019. Carnevale. La festa del mondo. Bari: Editori Laterza.

Merlo, Clemente. 1934. Studi glottologici. Pisa: Nistri-Lischi.

Nascimbente, Bruno, Francesco Rossi dal Pozzo. 2019. “L'evoluzione dei diritti e delle libertà fondamentali nel settore dei media. Diritto dell'Unione europea e orientamenti giurisprudenziali". Rivista.eurojus.it 4: 135-138.

Pasquini, Dario. 2012. "La satira e la storia delle emozioni. Una relazione privilegiata?". Diacronie. Studi di storia contemporanea: La satira fa storia. Eventi, prattiche, linguaggi 3(11): 1-16.

\section{REFLECTIONS ABOUT THE RIGHT OF SATIRA AND ITS LIMITS}

\section{Abstract}

Satire is an extreme paradigm of freedom of expression, but uncertainties exist about its conceptual definition and related legal regime. The development of communication has produced numerous literary figures similar to each other such as the chronicle (an impersonal and non-interpretive recording of events that have occurred), criticism (subjective analysis and judgment relating to events that have occurred) and satire (sarcastic criticism of characters, behaviors and actions 
of individuals for the purpose of social denunciation). The elements that characterize the satire, developed over the centuries, are essentially twofold: attention to contradictions (of politics, society, religion, culture) and moralistic intent to promote social change. Religious satire affects ecclesiastical power and its contradictions, but it also affects religious symbols and the contents of religions. Different legal consequences follow. When it affects the patrimony of faith of believers it is not acceptable. Religious satire generates a kind of conflict between different constitutional values, that is, between the right to the free expression of thought and the right to the reputation and protection of religious feelings. The right to satire in general is recognized by legal systems (both international and national) as a subjective right of constitutional relevance, which derives from the freedom of expression and thought. In the Charter of Fundamental Rights of the European Union, thought, conscience and religion are homologous (as legal goods or as ethical values). Therefore, thought, conscience and religion cannot be in opposition to each other. Considerable uncertainties exist about the legal discipline of the right to satire, which can never offend the fundamental rights of the person, his dignity and his reputation. The Nice Charter has favored an orientation which considers the right of free expression in its broadest and most expansive form. However, the prevailing value of fundamental human rights has always been affirmed, which cannot be offended by the exercise of the right to satire. The strength of the right to satire consists in the recognition of its constitutional rank, but also in the limits it must have. The jurisprudence has elaborated the "formal" obligations, among which the most important are: continence and functionality.

Key words: satire; religion; human rights; limitations

Ttumaczenie własne autora

\section{UWAGI NA TEMAT PRAWA DO SATYRY I JEGO OGRANICZEŃ}

Streszczenie

Satyra jest skrajnym wyrazem wolności wyrażania opinii. Zarówno jej ujęcie konceptualne, jak i relewantny reżim prawny, nie są wolne od kontrowersji. Jedną z konsekwencji rozwoju komunikacji międzyludzkiej jest wypracowanie licznych rodzajów literackich, które przejawiają pewne podobieństwa. Do takich należą m.in. kronika (bezosobowy i pozbawiony interpretacji zapis zaistniałych zdarzeń), krytyka (subiektywna analiza i ocena odnosząca się do zaistniałych 
zdarzeń) i satyra (sarkastyczna krytyka osób lub indywidualnych zachowań czy sposobów postępowania, mająca na celu ich społeczne zdemaskowanie). Ukształtowane w ciągu wieków elementy charakteryzujące satyrę są zasadniczo dwa: skoncentrowanie na wewnętrznych sprzecznościach (polityki, społeczeństwa, religii, kultury) oraz intencja moralizatorska, mająca na celu pobudzenie przemian społecznych. Satyra religijna bierze na cel władze religijne i ich niekonsekwencję, odnosi się jednak również do symboli religijnych i religijnych wierzeń. Wiążą się z tym różne konsekwencje prawne. Satyra, która skierowana jest na dziedzictwo wiary, musi być uznana za nieakceptowalną. Każda satyra religijna wiąże się natomiast z konfliktem pomiędzy różnymi wartościami konstytucyjnymi, to jest pomiędzy prawem do wyrażania opinii oraz prawem do dobrego imienia i do ochrony uczuć religijnych. Generalnie rzecz ujmując, prawo do satyry jest uznawane przez systemy prawne (zarówno na poziomie międzynarodowym, jak i na poziomie krajowym) jako prawo podmiotowe o znaczeniu konstytucyjnym, które wynika z wolności wyrażania opinii i wolności myśli. Myśl, sumienie i religia - zgodnie z ujęciem przyjętym na przykład w Karcie Praw Podstawowych Unii Europejskiej - są wartościami homologicznymi (jako dobra prawne czy wartości o charakterze etycznym). Nie mogą więc być między sobą w sprzeczności. Istotne wątpliwości dotyczą natomiast przepisów odnoszących się do prawa do satyry, która nie może naruszać podstawowych praw osoby, jej godności czy jej dobrego imienia. Karta Praw Podstawowych przyczyniła się do ugruntowania tendencji polegającej na ujmowaniu prawa do wyrażania opinii w jego najszerszej, ekspansywnej formie. Konsekwentnie potwierdzane jest jednak również znaczenie praw człowieka, które nie mogą być naruszane w konsekwencji realizowania prawa do satyry. W krajowych porządkach prawnych znaczenie prawa do satyry wiąże się z uznaniem jego konstytucyjnej rangi, ale także z ograniczeniami, którym ono musi podlegać. W orzecznictwie wypracowano relewantne kryteria formalne, wśród których najważniejsze znaczenie posiadają: powściągliwość i funkcjonalność.

Słowa kluczowe: satyra; religia; prawa człowieka; ograniczenia 
